\title{
Variations in circadian heart rate in psychiatric disorders: theoretical and practical implications
}

\author{
This article was published in the following Dove Press journal: \\ ChronoPhysiology and Therapy \\ 17 April 2013 \\ Number of times this article has been viewed
}

\section{HG Stampfer \\ SB Dimmitt ${ }^{2}$}

'School of Psychiatry and Clinical Neurosciences, University of Western Australia, ${ }^{2} \mathrm{School}$ of Medicine and Pharmacology, University of Western Australia, Crawley, WA, Australia
Correspondence: Hans G Stampfer School of Psychiatry and Clinical Neurosciences, University of Western Australia, 35 Stirling Highway, Crawley, WA 6009 , Australia

Tel +6I893462l40

Email hans.stampfer@uwa.edu.au:
Background: Data are presented to demonstrate dimensions of variation in circadian heart rate in patients under treatment for a psychiatric disorder and to comment on their clinical relevance.

Method: Serial recordings of 24-hour heart rates were obtained from individuals under treatment for a psychiatric disorder and from healthy volunteers.

Results: The mean 24-hour heart rate can vary independently of the circadian rate pattern or "rate architecture." Sleep and waking heart rate can vary independently. Variations in circadian heart rate are state-dependent: broadly different clinical states are associated with distinctly different patterns of circadian heart rate, particularly during sleep.

Conclusion: Different regulatory mechanisms or pathways are involved in mediating different aspects of circadian heart rate. An analysis of circadian heart rate can contribute useful physiological adjunct information to psychiatric assessment and the monitoring of patient response to treatment.

Keywords: anxiety, depression, neuroendocrine regulation

\section{Introduction}

Twenty-four hour (24 h) or circadian heart rate (CHR) is of interest in Psychiatry because it is subject to circadian regulation, ${ }^{1}$ and circadian dysregulation, that is associated with so-called "biological shift" symptoms, is present in varying degrees in all serious psychiatric disorders. ${ }^{2-5}$ Hence, changes in CHR may give some indication of circadian dysregulation, and this applies particularly to heart rate during sleep, since "external" confounding influences are minimal during sleep. Today, studies of heart rate focus on heart rate variability, but there is no direct or consistent relationship between rate and rate variability, ${ }^{6,7}$ and the interest here is in heart rate measured in beats per minute (bpm).

Data are presented to show that broad differences in CHR within and between individuals can be defined systematically in terms of the $24 \mathrm{~h}$ mean, sleep-wake rate difference, and sleep-wake mean trend pattern. Each of these aspects can vary independently, and distinctly different combinations of variation can be found in different psychiatric disorders, as well as serially in state-dependent changes within individuals. Insofar as these variations, particularly during sleep, reflect differences in circadian neuroendocrine regulation, an analysis of CHR may contribute useful physiological adjunct information to psychiatric assessment, the selection of medication, and monitoring of patient response to treatment. 


\section{Method}

\section{Study participants}

Serial recordings of $24 \mathrm{~h}$ heart rate were obtained from healthy volunteers and in-patients under treatment for a primary psychiatric disorder diagnosed according to DSM-IV criteria (Diagnostic and Statistical Manual of Mental Disorders, fourth edition, American Psychiatric Association). The following criteria applied to individuals selected for heart rate monitoring: age 17-50 years; physically healthy with no history or evidence of heart disease; not abusing alcohol or illicit drugs; and not taking prescribed drugs with a recognized effect on heart rate (eg, amphetamines, beta blockers, thyroxine). Patients taking prescribed anxiolytic, antidepressant, or antipsychotic drugs (apart from clozapine) were not excluded.Recordings from patients were obtained 1-2 days after admission (to a psychiatric unit at a general hospital) and 1-2 day prior to discharge - in case of longer admissions, at 1-2 weekly intervals. Serial recordings from healthy volunteers were obtained at an interval of around 2 weeks.

The presented examples of variations in CHR were taken from investigations conducted over several years since 1994. Over 1500 patients (representing all commonly encountered psychiatric disorders) were monitored along with over 300 healthy volunteers. Patients gave informed consent to have their heart rate monitored under supervision of their treating psychiatrist and formal approval for a study on heart rate was obtained from a hospital ethics committee (QEII Medical Centre).

\section{Data acquisition - recording of heart rate and activity}

The monitor used to obtain heart rate (Leigh Mardon Systems, NSW, Australia) identifies "R" waves and stores the number occurring every minute. Activity was monitored in parallel with heart rate to control for activity-related changes in heart rate. Heart rate monitoring was undertaken in conjunction with a mental state examination by a senior psychiatrist.

\section{Results}

\section{Variation in the mean trend pattern during sleep}

Heart rate patterns during sleep can vary widely between individuals, especially in individuals suffering from serious forms of mental illness. Two patterns of interest here are the "mirror-opposite" deviations from the typically normal sleep-wake rate pattern or "architecture," as illustrated in Figure 1.

Figure 1 shows $24 \mathrm{~h}$ recordings of heart rate from three male subjects identified as "A," "B," and "C." Subject A, a 35-year-old, was taking paroxetine $20 \mathrm{mg}$ daily and diazepam $5 \mathrm{mg}$ twice daily for social phobia and generalized anxiety disorder (GAD), whereas subject $\mathrm{B}$, a 30-year-old, was physically fit with no medical or psychiatric history, and subject $\mathrm{C}$, a 31-year-old, was taking sertraline $150 \mathrm{mg}$ daily for "agitated" melancholic depression.

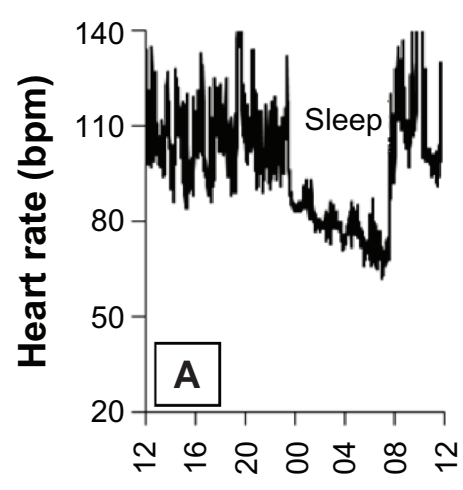

A

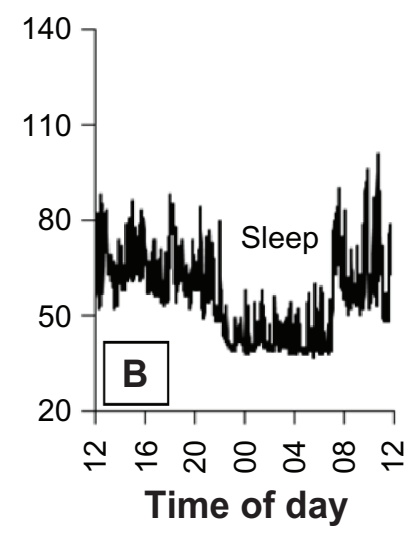

$B$

64

38

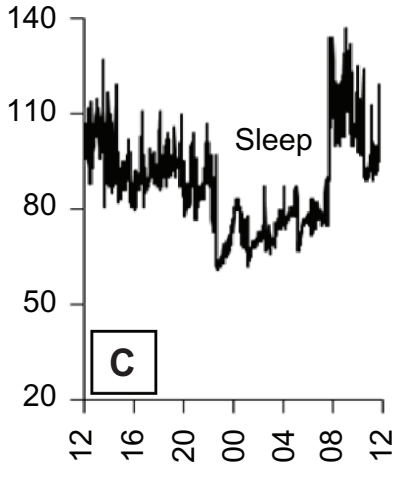

C

88

74

Figure I The images show $24 \mathrm{~h}$ recordings of heart rate from three males identified as (A-C).

Notes: The pattern for subject B was "signature" normal, ie, similar to that typically found in physically fit, well-adjusted individuals leading a stable life. Subjects A and C showed mirror-opposite mean trend patterns during sleep that were seemingly a manifestation of differences in neurendocrine regulation. Sleep intervals are indicated. Twenty-four hour and sleep means are indicated below each plot.

Abbreviations: $24 \mathrm{~h}$, twenty-four hour; bpm, beats per minute. 
The pattern for subject B was similar to that typically found in physically fit, well-adjusted individuals leading a stable life. It showed a clearly defined sleep-wake rate architecture with a rectangular pattern of sleep related changes. Both the onset of sleep and waking showed an abrupt change in rates, and there was a fairly constant lower rate during sleep except for periodic (rapid eye movement [REM]-sleep related) increases in rate variability. The $24 \mathrm{~h}$ mean and sleep mean were well within normal limits. Subjects A and C showed mirror-opposite mean trend patterns during sleep that were not attributable to differences in activity, fitness, medications, or any other external factor and were seemingly a manifestation of differences in neurendocrine regulation.

\section{Pattern similarity between individuals across wide variation in $24 \mathrm{~h}$ mean}

Comparisons of data between individuals show that closely similar circadian patterns can persist independently of the $24 \mathrm{~h}$ mean, as illustrated in Figure 2.

The data shown in Figure 2 were obtained from four female patients identified as "D," "E," "F," and "G." Subject D, a 24-year-old, was treated for a schizoaffective disorder with quetiapine $600 \mathrm{mg}$ daily and venlafaxine $225 \mathrm{mg}$ daily. Subject E, a 28-year-old, was taking paroxetine $20 \mathrm{mg}$ daily for GAD. Subject F, a 36-year-old, was treated for psychotic depression with mirtazepine $60 \mathrm{mg}$ at night and olanzapine $10 \mathrm{mg}$ at night. Subject G, a 30-year-old, was treated for major depression with sertraline $100 \mathrm{mg}$ daily. The data were grouped in pairs, based on the similarity of the mean trend pattern during sleep. The data from subjects D and E data showed a similar downward trend or "down-ramp" sleep pattern, while the data from subjects $F$ and $G$ data showed a similar upward trend or "up-ramp" sleep pattern. Despite the pattern similarity in each pairing, the $24 \mathrm{~h}$ mean differed by $39 \mathrm{bpm}$ in each pairing. Only two examples were shown in Figure 2, but a continuum of variation was found across a wide range of $24 \mathrm{~h}$ means. This suggests that rate and circadian rate architecture involve broadly different regulatory mechanisms that can vary independently. Direct evidence of this was found in serial within-individual data, as illustrated in Figure 3.

\section{Persistence of the circadian pattern within individuals, independently of a variation in $24 \mathrm{~h}$ mean}

The data in Figure 3 were obtained from two patients identified as "H" and "I." For these patients, the recordings were obtained two weeks apart. Subject H, a 29-year-old female with a severe borderline personality disorder, was admitted for treatment of a paranoid delusional state that had developed after smoking marijuana. She was taking olanzapine $10 \mathrm{mg}$ daily at the time of the first recording and $20 \mathrm{mg}$ daily at the time of the second. Subject I, a 36-year-old female with a history of recurrent depression, was treated for a further episode of depression. She was taking sertraline $100 \mathrm{mg}$ daily at the time of the first recording and sertraline $100 \mathrm{mg}$ plus clonazepam $1 \mathrm{mg}$, twice daily at the time of the second recording. The serial change in the data, for both $\mathrm{H}$ and I, was predominantly a lowering of the $24 \mathrm{~h}$ mean but with preservation of a closely similar circadian pattern, particularly during sleep. The data from subject $\mathrm{H}$ showed a decrease of $14 \mathrm{bpm}$ in the $24 \mathrm{~h}$ mean, whereas the data from subject I
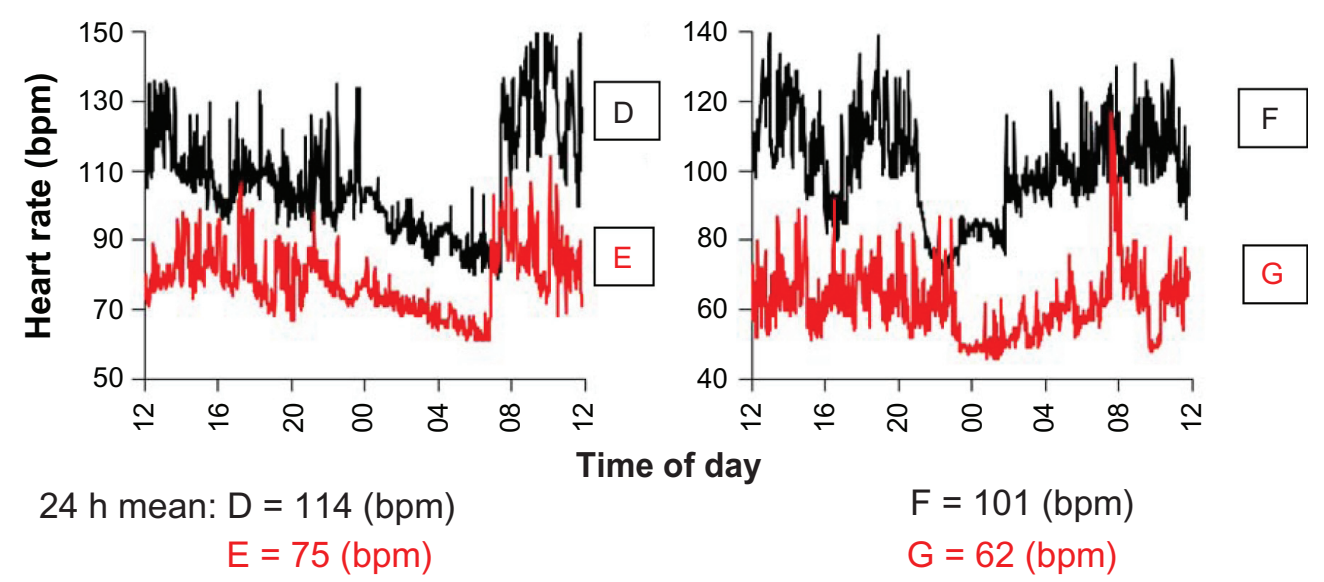

Figure 2 Comparisons of data between paired patients (D-G) show that closely similar circadian patterns can persist, independently of the 24 h mean. Notes: The data were grouped in pairs, based on the similarity of the mean trend pattern during sleep. Twenty-four hour means are indicated below each plot. Abbreviations: $24 \mathrm{~h}$, twenty-four hour; bpm, beats per minute. 

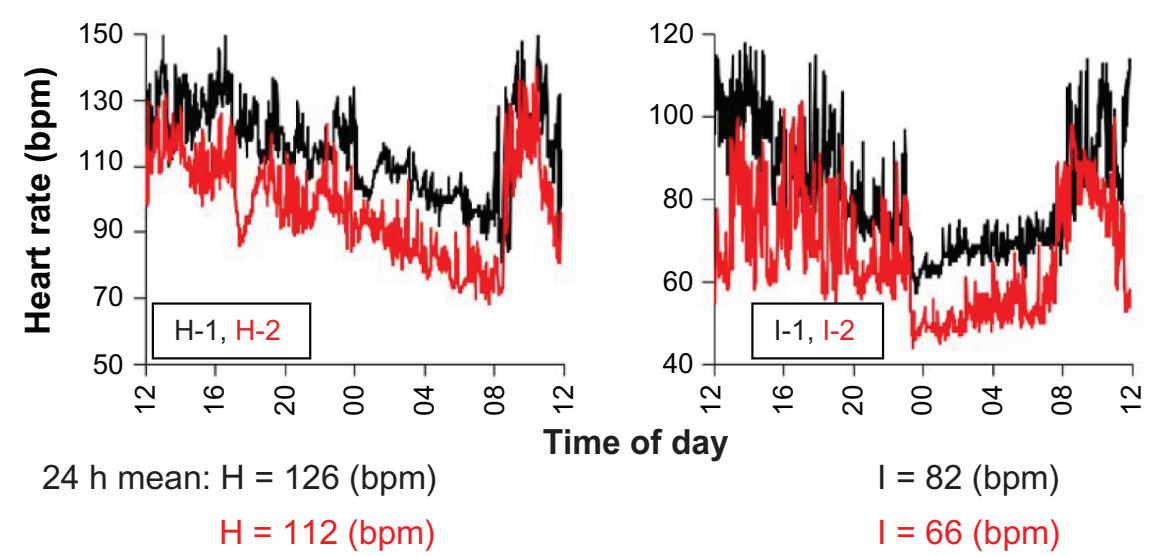

Figure 3 The images show recordings from two patients, $(\mathbf{H}$ and $\mathbf{I})$.

Notes: For each patient, the recording was repeated after a 2-week interval. The noted change was predominantly a variation in the mean, with preservation of the circadian pattern. Twenty-four hour means are indicated below each plot.

Abbreviations: $24 \mathrm{~h}$, twenty-four hour; bpm, beats per minute.

showed a decrease of $12 \mathrm{bpm}$. Serial within-individual changes usually involve variation in more than one aspect of circadian heart rate, but as illustrated in Figure 3, the change can be predominantly a variation in the mean, with preservation of the circadian pattern.

\section{Combined down-up ramp pattern during sleep}

Some individuals reveal sleep patterns that appear to be a combination of the down-ramp and up-ramp patterns shown in Figures 1-3. Two typical examples are given in Figure 4, showing that these patterns can also persist over a wide variation in the $24 \mathrm{~h}$ mean.

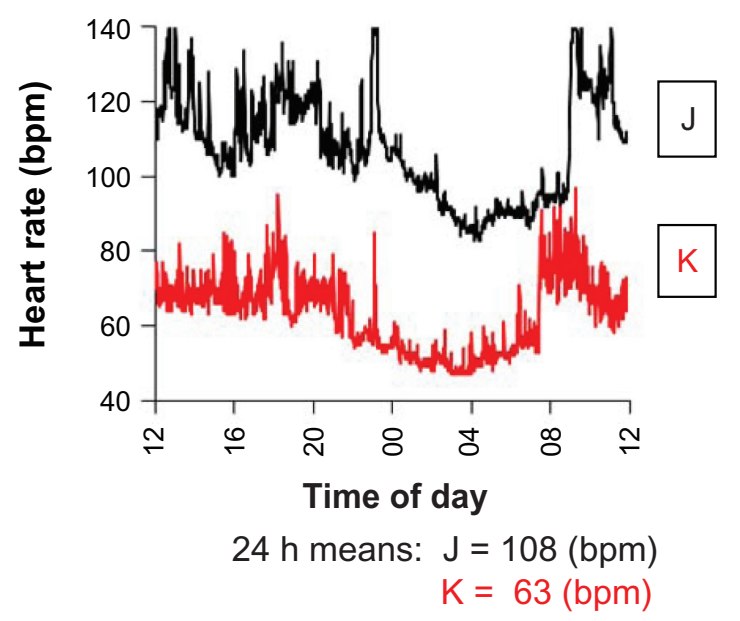

Figure 4 Data from two female patients identified as ( $\mathbf{J}$ and $\mathbf{K}$ ) showed a combination of down-ramp and up-ramp patterns that persisted over a wide variation in the $24 \mathrm{~h}$ mean.

Note: The twenty-four hour means are indicated below the plot. Abbreviations: $24 \mathrm{~h}$, twenty-four hour; bpm, beats per minute.
The data in Figure 4 were obtained from two female patients identified as "J" and "K." Subject J was a 35-year-old woman with a severe borderline personality disorder and a long history of repeated hospital admissions for self-harm and brief psychotic episodes. She was taking quetiapine $500 \mathrm{mg}$ daily and escitalopram $40 \mathrm{mg}$ daily. Subject K was a 31-year-old woman under treatment for double depression (dysthymia and major depression), with mirtazepine $30 \mathrm{mg}$ at night. As illustrated in Figure 4, there was variation in the gradient and duration of the down- and up-ramp trend, but the change from downward to upward occurred during sleep in these subjects and was not related to activity or other external factor.

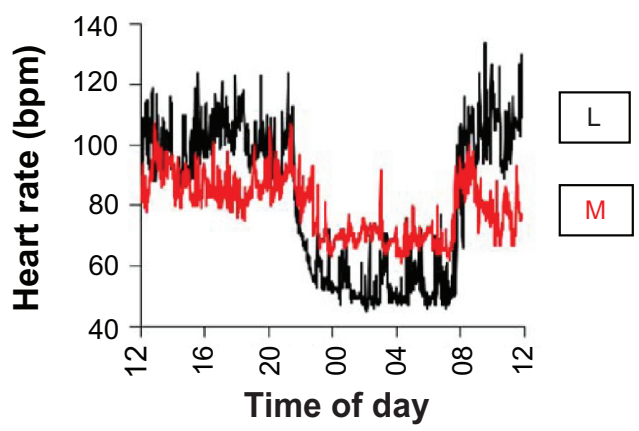

\begin{tabular}{rll} 
& \multicolumn{1}{c}{$\mathbf{L}$} & \multicolumn{1}{c}{$\mathbf{M}$} \\
24 h mean: & $82(\mathrm{bpm})$ & $79(\mathrm{bpm})$ \\
Waking mean: & $101(\mathrm{bpm})$ & $85(\mathrm{bpm})$ \\
Sleep mean: & $55(\mathrm{bpm})$ & $69(\mathrm{bpm})$ \\
Sleep-wake $\Delta:$ & $46(\mathrm{bpm})$ & $16(\mathrm{bpm})$
\end{tabular}

Figure $\mathbf{5}$ Data obtained from two male patients identified as ( $\mathbf{L}$ and $\mathbf{M})$ showed that there can be wide variation in the sleep-wake rate difference.

Note: The twenty-four hour, waking, and sleep means are indicated below the plot. Abbreviations: $24 \mathrm{~h}$, twenty-four hour; bpm, beats per minute. 


\section{Between-individual variations in the sleep-wake rate difference}

Between-individual comparisons show that there can be wide variation in the sleep-wake rate difference, as illustrated in Figure 5.

The data in Figure 5 were obtained from two male patients identified as "L" and "M." Subject L, a 28-yearold, was under treatment for a paranoid delusional disorder. At the time of recording, he harbored overvalued but not frankly delusional ideas. His only medication was risperidone $25 \mathrm{mg}$ intramuscularly (IM) every 2 weeks. Subject $\mathrm{M}$, a 32-year-old, was taking clomipramine $150 \mathrm{mg}$ at night for treatment of an obsessive-compulsive disorder. He had responded well and was due for discharge at the time of recording. Comparing the two records, it was seen that while the $24 \mathrm{~h}$ mean differed by only $3 \mathrm{bpm}$, the sleep-wake rate difference for subject $\mathrm{L}$ was $46 \mathrm{bpm}$, whereas for subject $\mathrm{M}$ this was $16 \mathrm{bpm}$. Variation in the sleep-wake rate difference was also evident in the serial within-individual data, and serial data show that variation can occur independently in the sleep and waking state. Examples of this are given in Figure 6.

\section{Independent variation in the sleep and waking state within individuals}

Figure 6 shows superimposed serial recordings from two male patients identified as "N" and "O." Subject N, a 28-year-old, developed a mild delusional state in a context of stressful family circumstances. He was treated with olanzapine $10 \mathrm{mg}$ at night and was no longer delusional by the time of the second recording. Superimposed serial recordings, taken at admission and one week later, showed a closely similar mean trend during sleep, with sleep means differing by an insignificant $2 \mathrm{bpm}$. In contrast, waking recordings showed a significant mean difference of $18 \mathrm{bpm}$ from midday to sleep and a large difference of $31 \mathrm{bpm}$ from waking to midday. Subject $\mathrm{O}$, a 36-year-old, was taking fluoxetine $20 \mathrm{mg}$ daily for dysthymia. For subject $\mathrm{O}$, the serial recordings taken on admission and 8 days later showed waking means that did not differ significantly, but the sleep means showed a significant difference of $14 \mathrm{bpm}$. Serial within-individual recordings usually varied both in the sleep and waking state but, as shown in these examples, significant variation could be largely confined to the sleep or waking state.

\section{State-dependent serial changes}

Serial recordings from individuals under treatment for a psychiatric disorder can show evidence of no significant change, worsening (greater "deviation" from the signature normal pattern), or improvement (closer approximation to the signature normal pattern) in the circadian pattern. Examples of "no change" and "worse" are shown in Figure 7.
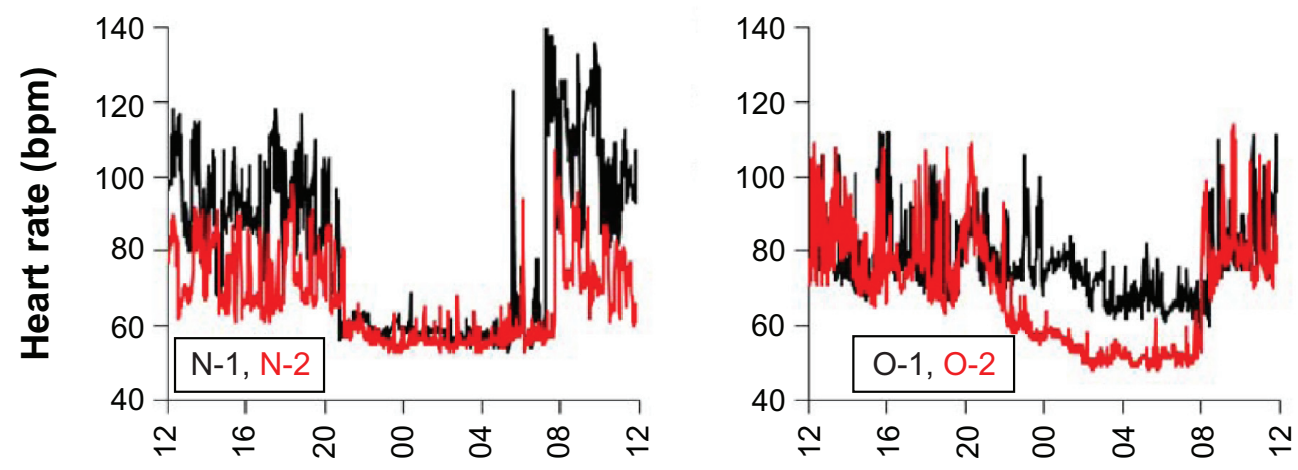

Time of day

$\begin{array}{cccc} & \text { PM } & \text { Sleep } & \text { AM } \\ \mathrm{N}-1 & 92 & 59 & 107 \\ \mathrm{~N}-2 & 74 & 57 & 76 \\ \Delta & -18 & 2 & 31\end{array}$

$\begin{array}{cccc} & \text { PM } & \text { Sleep } & \text { AM } \\ \text { O-1 } & 79 & 69 & 84 \\ \text { O-2 } & 81 & 55 & 81 \\ \Delta & 2 & -14 & -3\end{array}$

Figure 6 The images show superimposed serial recordings from two male patients identified as ( $\mathbf{N}$ and $\mathbf{O})$.

Notes: Midday to sleep (PM), sleep, and waking to midday (AM) means (and the difference between the two recordings " $\Delta$ ") are indicated below each figure. For ( $\mathbf{N}$ ), the first recording (black) was taken the day after he was admitted; the second (red) one week later; for (O), the first recording (black) was taken the day after he was admitted; the second (red) 8 days later.

Abbreviation: bpm, beats per minute. 
"No change"

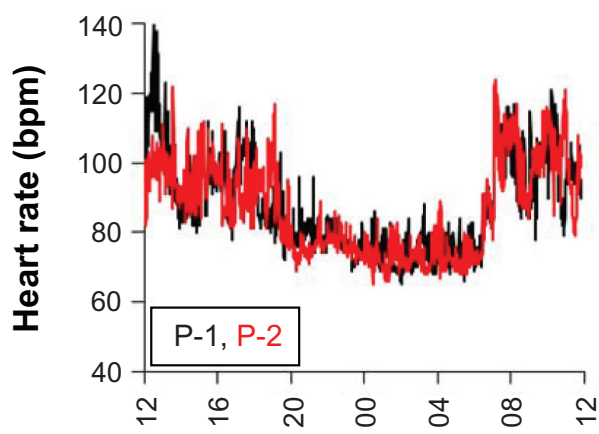

"Worse"

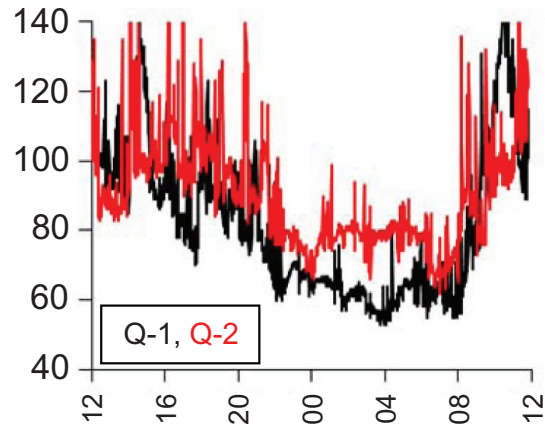

\section{Time of day}

$\begin{array}{rcccc} & \text { P-1 } & \text { P-2 } & \text { Q-1 } & \text { Q-2 } \\ \text { 24 h mean: } & 87 & 87 & 82 & 90 \\ \text { Sleep mean: } & 76 & 74 & 63 & 77\end{array}$

Figure 7 Two superimposed serial recordings from two individuals showed $(\mathbf{P})$ no change; and $(\mathbf{Q})$ worsening, in the circadian pattern.

Notes: The twenty-four hour and sleep means are indicated below each plot. For (P), the first recording (black) was taken the day after he was admitted; the second (red), 6 days later. For (Q), the first recording (black) was taken the day after he was admitted; the second (red), 2 weeks later. He reported feeling more depressed at the time of the second recording, which shows an increase in the $24 \mathrm{~h}$ mean and sleep mean, as well as a more disorganized circadian pattern.

Abbreviations: $24 \mathrm{~h}$, twenty-four hour; bpm, beats per minute.

Figure 7 shows two superimposed serial recordings from two subjects identified as "P" and "Q." Subject P, a 27-year-old female with a diagnosis of borderline personality disorder was admitted after an episode of self-harm (forearm cutting that required sutures) following interpersonal conflict. Two recordings from this subject, taken on the day after admission and 6 days later, showed a closely similar circadian pattern, an identical $24 \mathrm{~h}$ mean, and practically the same sleep mean.

Subject Q was a 25-year-old man with a diagnosis of Cluster B personality disorder (narcissistic and borderline traits) who was being treated for depression with escitalopram $40 \mathrm{mg}$ daily. The first recording on this subject was obtained the day following his admission, the second
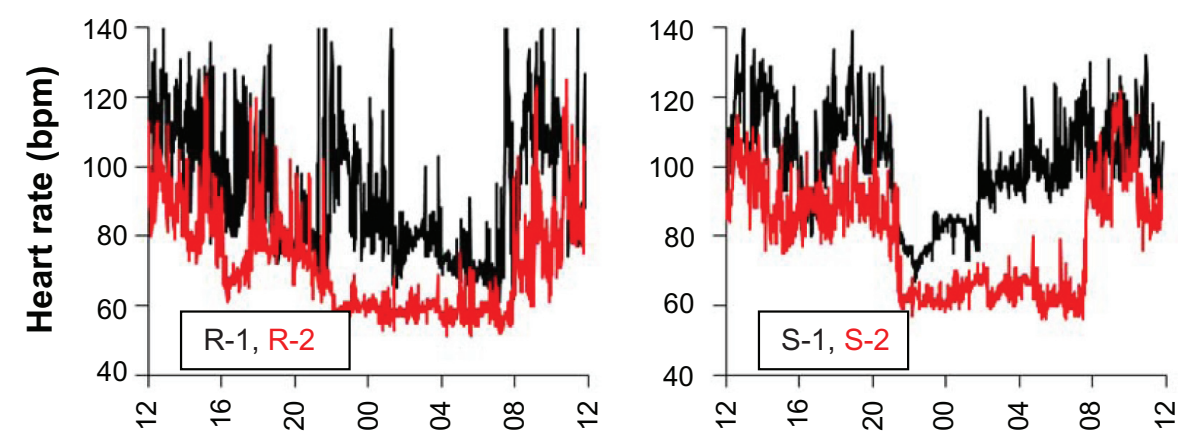

\section{Time of day}

$\begin{array}{rcccc} & \text { R-1 } & \text { R-2 } & \text { S-1 } & \text { S-2 } \\ \text { 24 h mean: } & 92 & 74 & 101 & 80 \\ \text { Sleep mean: } & 72 & 59 & 79 & 63\end{array}$

Figure 8 Two serial recordings from two individuals, ( $\mathbf{R}$ and $\mathbf{S}$ ), suggested that the more effective the treatment, the greater evidence of normalization of the circadian pattern.

Notes: The twenty-four hour and sleep means are indicated below each plot. For (R), the second recording (red) was taken 10 days after the first recording (black). The $24 \mathrm{~h}$ mean has lessened by $18 \mathrm{bpm}$, the sleep mean by $13 \mathrm{bpm}$, and the circadian sleep-wake rate architecture resembled the pattern found in healthy, well-adjusted individuals without a history of psychiatric illness. For (S), the second recording (red) was taken three weeks after the first (black). The $24 \mathrm{~h}$ mean had lessened by 21 bpm, the sleep mean by $16 \mathrm{bpm}$, and the circadian pattern had normalized.

Abbreviations: 24 h, twenty-four hour; bpm, beats per minute. 
2 weeks later. He reported feeling more depressed at the time of the second recording, which showed in increase in the $24 \mathrm{~h}$ mean and sleep mean, as well as a more disorganized circadian pattern.

\section{State-dependent serial changes: improvement/normalization}

Serial recordings suggested that the more effective the treatment, the more "normalization" of the circadian pattern. This is illustrated in Figure 8.

Figure 8 shows two serial recordings from two subjects identified as "R" and "S." In both of these cases, treatment was very effective. Subject R, a 35-year-old man, was treated for severe GAD following a highly stressful event. His medications included diazepam $5 \mathrm{mg}$ four times daily and temazepam $20 \mathrm{mg}$ at night. The second recording for this subject was taken 10 days after the first recording. It showed that the $24 \mathrm{~h}$ mean had lessened by $18 \mathrm{bpm}$, the sleep mean by $13 \mathrm{bpm}$, and the circadian sleep-wake rate architecture had normalized, in that it closely resembled the pattern found in healthy, well-adjusted subjects without a history of psychiatric illness.

Subject "S," a 36-year-old female, was treated for psychotic depression with mirtazepine $60 \mathrm{mg}$ at night and olanzapine $10 \mathrm{mg}$ at night. Three weeks after the first recording, the second recording was taken and showed that the $24 \mathrm{~h}$ mean had lessened by $21 \mathrm{bpm}$, the sleep mean by $16 \mathrm{bpm}$, and the circadian pattern had normalized.

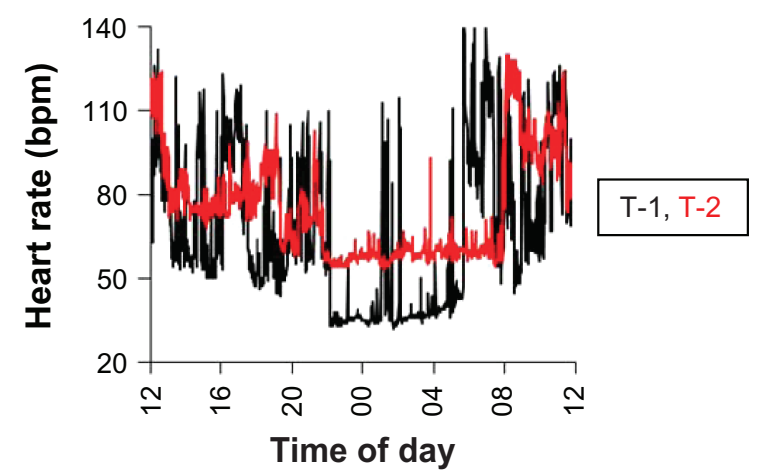

$\begin{array}{rccc} & \text { T-1 } & \text { T-2 } & \Delta \\ \text { 24 h mean: } & 68 & 75 & 7 \\ \text { Waking mean: } & 79 & 87 & 8 \\ \text { Sleep mean: } & 40 & 59 & 19\end{array}$

Figure 9 Superimposed serial recordings obtained 6 weeks apart from a 25-yearold man treated for psychotic depression showed normalization with an increase in rates, particularly during sleep.

Notes: The twenty-four hour, waking, and sleep means are indicated below the plot. $\Delta$ is the difference between the two recordings.

Abbreviations: 24 h, twenty-four hour; bpm, beats per minute.
Two examples of normalization have been described to show that despite significantly different patterns before treatment, effective treatment resulted in a similar, typically normal pattern. The majority of patients do not show normalization to such an extent, but the more there is evidence is of normalization, the better the results of treatment. Normalization is usually associated with a lessening of abnormally elevated rates, but occasionally the reverse occurs, as illustrated in Figure 9.

Figure 9 shows superimposed serial recordings obtained 6 weeks apart, from a 25-year-old man who was treated for psychotic depression with sertraline $150 \mathrm{mg}$ daily and olanzapine $15 \mathrm{mg}$ daily. His symptoms had remitted fully at the time of the second recording. The normalization of the circadian pattern in this case included a large increase in the sleep mean from 40 to $59 \mathrm{bpm}$, a lesser increase in the waking mean, and an increase in sleep time from 6.5 hours to 10 hours. Unlike the normalization in Figure 8, which showed a reduction in rates, the normalization in Figure 9 showed an increase in rates, particularly during sleep.

\section{Discussion}

The presented data demonstrate dimensions of variation in $\mathrm{CHR}$ that have not been reported previously. Differences in CHR within and between individuals can be described systematically in terms of the variation in these different aspects of 24-hour activity. Evidence that these different aspects of activity can vary independently suggests the involvement of different regulatory mechanisms or pathways, whose identification may help to better understand physiological differences between psychiatric disorders. The wide variation in CHR across the spectrum of classified psychiatric disorders cannot be explained by fitness, age, gender, activity, or psychotropic medications, either singly or in combination. Physically healthy individuals closely matched on these variables but suffering from broadly different forms of mental illness such as GAD and melancholic depression, show distinctly different patterns of CHR so as to suggest that pattern differences are predominantly a manifestation of physiological differences between these disorders.

Previous work reported that broadly different psychiatric disorders are associated with distinctly different circadian patterns. ${ }^{8}$ Further data since then, from a large number of patients representing a broad spectrum of DSM-IV disorders, have confirmed that this is indeed the case. However it is now also apparent that whilst some classified disorders, for example melancholic depression, are consistently associated with distinctive changes in CHR, others, for example, 


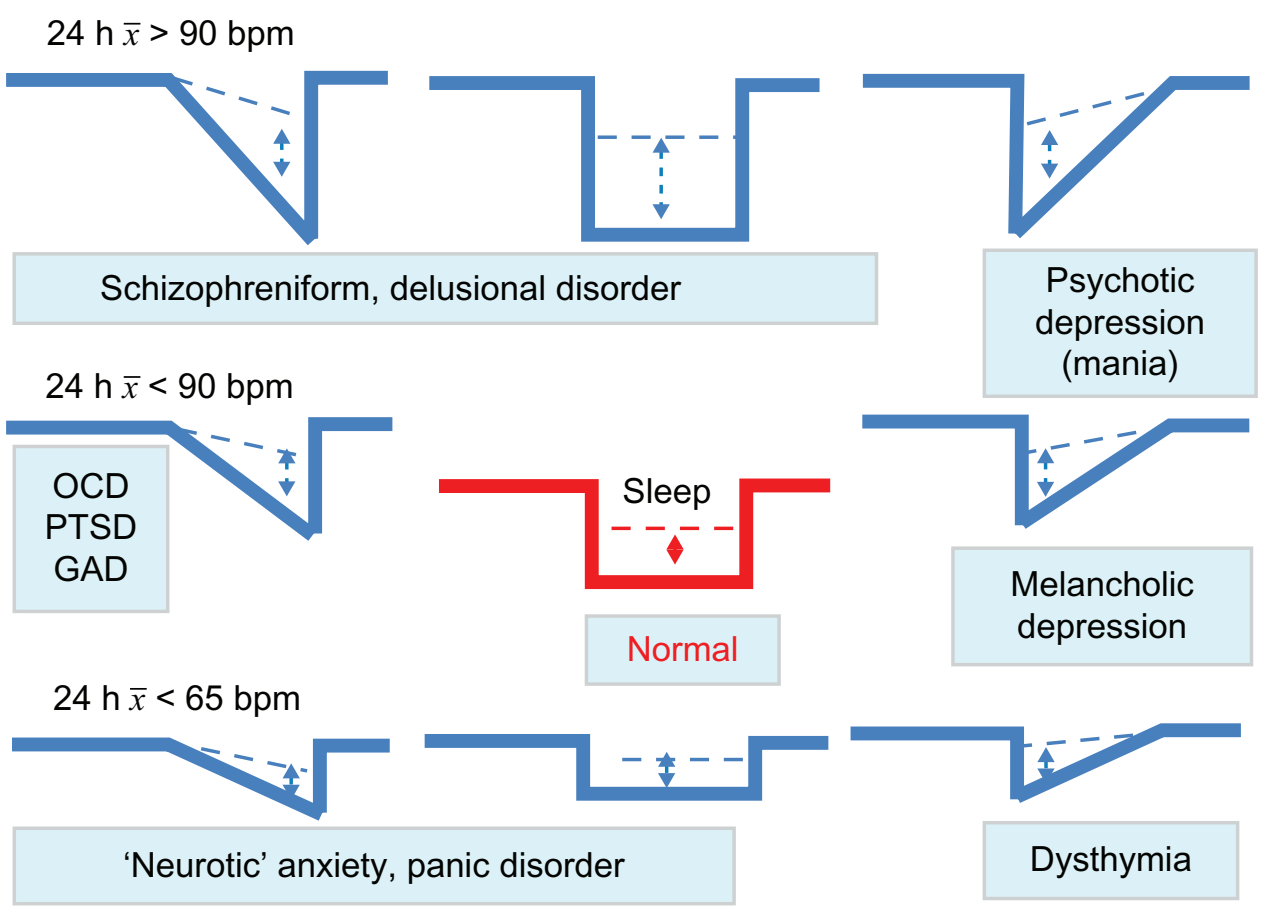

Figure 10 Simplified diagrammatic overview of commonly observed relationships between broadly different patterns of CHR and psychiatric status. Abbreviations: bpm, beats per minute; CHR, circadian heart rate; GAD, generalized anxiety disorder; OCD, obsessive-compulsive disorder; PTSD, posttraumatic stress disorder.

the somatoform disorders as a group, are not. This would suggest that insofar as CHR is any indication, melancholic depression is a relatively distinct entity, whereas somatoform disorders are not. Even then, indication of depression in CHR will not necessarily predict the clinical manifestations because depression can present in different clinical guises. ${ }^{9-12}$ The same uncertainty in this regard would apply to every biological marker. The more consistently observed relationships between CHR and psychiatric status are summarized in Figure 10.

Figure 10 is a simplified diagrammatic overview of commonly observed relationships between broadly different patterns of CHR and psychiatric status and shows that the deviations from normal can be broadly classified as "downramp," "up-ramp," "low rate" ( $24 \mathrm{~h}$ mean $<65 \mathrm{bpm}$ ), and "high rate" ( $24 \mathrm{~h}$ mean $>90 \mathrm{bpm}$ ). High rate patterns often involve widening of the sleep-wake rate difference, but narrowing (and even sleep rates higher than waking rates) can also be found.

The down-ramp patterns are broadly associated with anxiety, the up-ramp patterns with depression, low rate patterns with neurotic disorders, high rate patterns with psychotic disorders. Psychotic disorders are usually associated with high rate patterns, but severe/psychotic depression may sometimes be associated with an abnormally low rate pattern, as was illustrated above in Figure 9.
The mirror-opposite sleep mean trend patterns in anxiety and depression suggest an opposite balance of sympathetic and parasympathetic nervous system activity, since the autonomic nervous system plays a prominent role in the regulation of heart rate. ${ }^{13}$ In particular, the down-ramp pattern suggests a dominance of sympathetic activity at the onset of sleep, followed by a progressive decline in dominance until waking, whereas the up-ramp pattern suggests a dominance of parasympathetic activity at the onset of sleep, followed by a progressive decline in dominance and possibly increased sympathetic activity that may contribute to early morning waking, typically associated with melancholic depression.

Variation in the sleep mean trend is not limited to downramp, "horizontal," up-ramp, and combined down-up ramp (as shown in Figures 1 and 2), but a discussion of the wider variation encountered is beyond the scope of this article.

Heart rate data show that a similar circadian pattern may be associated with different clinical diagnoses, and a good example of diagnostic overlap on the same pattern is seen in Figure 10, where the same medium-rate (24 h mean $<90 \mathrm{bpm}$ ) down-ramp pattern was associated with clinical diagnoses of GAD, obsessive-compulsive disorder (OCD) and posttraumatic stress disorder - to which could be added "acute stress disorder" and "adjustment disorder with anxious mood." All these disorders may show a similar pattern of CHR, suggesting a much the same physi- 
ological "shift," despite variation in the clinical manifestations. What is perhaps more interesting from a classificatory perspective, is that a major change in the $24 \mathrm{~h}$ mean within a similar circadian pattern, which seems a purely quantitative change at a physiological level, can result in widely different clinical manifestations, as indicated for example, in the recordings for schizophreniform and delusional disorder, and neurotic, anxiety, and panic disorder, in Figure 10. This is somewhat analogous to a change in temperature altering the manifestations of water from ice, to liquid to steam. The changing manifestations of increases in the $24 \mathrm{~h}$ mean depend on the circadian pattern: in this set of disorders, an elevation of the down-ramp pattern culminated in schizophreniform manifestations, whereas elevation of the up-ramp pattern culminated in psychotic depression.

It has long been recognized that mood disorders are associated with sleep and circadian abnormalities, ${ }^{2}$ and similar abnormalities are also found in other psychiatric disorders. ${ }^{5}$ Evidence is emerging that the effective treatment of mood disorders involves normalization of circadian rhythms, ${ }^{4}$ and evidence of this is suggested in the serial recordings of CHR. Figures 8 and 9 show examples of effective treatment that resulted in a similar normal circadian pattern in different individuals with distinctly different pretreatment patterns. In contrast, the examples in Figure 7 showed that when treatment was ineffective, the circadian pattern remained much the same or worsened. Serial data from patients treated for a broad spectrum of classified disorders suggest that the greater the evidence of normalization towards the typical normal pattern (as shown in Figures 8 and 9), the more effective the treatment. Often treatment does not achieve this degree of normalization, so as to suggest only partial effectiveness and/or underlying trait disorders that are also associated with changes in CHR. Evidence of state-dependent changes, in which individuals acted as their own control, suggests that changes in CHR are part of the physiological shift in psychiatric disorders and that distinctly different circadian patterns, particularly those occurring during sleep, are manifestations of different physiological shifts. That being the case, CHR can add practically useful information to diagnostic assessment and to the monitoring of patient response to treatment.

From a wider perspective, the inclusion of the circadian perspective in studies of heart rate can be expected to provide additionally useful information. There is no mention of the circadian aspect in the considerable literature on resting heart rate and all-cause morbidity/mortality. ${ }^{14-17}$ However, neither resting heart rate nor the $24 \mathrm{~h}$ mean is a reliable predictor of heart rate during sleep, and the potentially wide variation between individuals in sleep rates/rate pattern (despite the same resting heart rate or $24 \mathrm{~h}$ mean) may be confounding to findings based purely on measures of resting heart rate during waking hours. Studies of resting heart rate should control for possible variation in the sleep-wake rate difference between individuals, since there may be wide variation in this regard.

\section{Limitations}

Single recordings are necessary and sufficient to show variation in CHR, but further studies are required to substantiate the idea proposed here, namely, that changes in CHR are part of the biological shift in psychiatric disorders. Only a descriptive account has been given here, and it is not known to what extent variations in CHR reflect specific biological changes in different disorders. The strongest diagnostic distinction in terms of CHR was found between broadly defined anxiety and depression, as indicated by the mirror-opposite sleep mean trend patterns discussed above. There was also a strong association between very high rate patterns and psychosis that did not merely reflect nonspecific arousal, since distinctly different high rate patterns were associated with different psychotic manifestations. Recognizing that further studies are required, there is justification for suggesting that the distinctly different CHR patterns in different psychiatric disorders are, to some extent, a reflection of physiological differences between those disorders.

The idea of a "deviation" in CHR is only meaningful in relation to a normal reference, and whilst there is a sizeable literature on normal resting and average heart rate, ${ }^{14,18,19}$ there are only isolated studies ${ }^{20}$ on the normal variation in circadian rate architecture, particularly with respect to sleep-related changes. Cosinor analysis has been used to describe circadian variation in heart rate, ${ }^{21}$ but this approach does not adequately account for differences in sleep-related rate changes or the dimensions of variation described here. The objective delineation of the normal variation in sleeprelated rate architecture poses a number of problems and remains a work in progress. Earlier, it was suggested that the recordings of subject B in Figure 1 exemplified a "signature" normal pattern: The $24 \mathrm{~h}$ and sleep means were well within normal limits, the sleep-wake rate difference was greater than $10 \mathrm{bpm}$, and, insofar as lower sleep rates are desirable from a physiological perspective, the rectangular pattern of sleep-wake rate architecture suggested optimal regulatory changes. This pattern is typically found in healthy, physically fit adults who do not abuse alcohol or drugs. It is, in this sense, at one end of a continuum of variation that spans 
the widely divergent patterns found in different individuals, particularly in those suffering from serious forms of mental illness. The task remaining is to objectively define the normal variation in sleep-wake rate architecture.

\section{Disclosure}

The authors report no conflicts of interest in this work.

\section{References}

1. Minors DS, Waterhouse JM. Circadian Rhythms and the Human. London: John Wright; 1981.

2. Howland RH, Thase ME. Affective disorders: biological aspects. In: Millon T, Blaney PH, Davis RD, editors. Oxford Textbook of Psychopathology. New York, NY: Oxford University Press; 1999:166-202.

3. Monteleone P, Maj M. The circadian basis of mood disorders: recent developments and treatment implications. Eur Neuropsychopharmacol. 2008;18(10):701-711.

4. Wirz-Justice A, Bromundt V, Cajochen C. Circadian disruption and psychiatric disorders: the importance of entrainment. Sleep Med Clin. 2009;4(2):273-284.

5. Wulff K, Gatti S, Wettstein JG, Foster RG. Sleep and circadian rhythm disruption in psychiatric and neurodegenerative disease. Nat Rev Neurosci. 2010;11(8):589-599.

6. Abildstrom SZ, Jensen BT, Agner E, et al; BEAT Study Group. Heart rate versus heart rate variability in risk prediction after myocardial infarction. J Cardiovasc Electrophysiol. 2003;14(2):168-173.

7. Coumel P, Maison-Blanche P, Catuli D. Heart rate and heart rate variability in normal young adults. $J$ Cardiovasc Electrophysiol. 1994;5(11):899-911.

8. Stampfer HG. The relationship between psychiatric illness and the circadian pattern of heart rate. Aust N Z J Psychiatry. 1998;32(2): 187-198.

9. Kirmayer LJ. Cultural variations in the clinical presentation of depression and anxiety: implications for diagnosis and treatment. J Clin Psychiatry. 2001;62 Suppl 13:22-28.
10. Şar V, Akyüz G, Kundakçı T, Kızıltan E, Dogan O. Childhood trauma, dissociation and psychiatric comorbidity in patients with conversion disorder. Am J Psychiatry. 2004;161(12):2271-2276.

11. Khan MN, Ahmad S, Arshad N, Ullah N, Maqsood N. Anxiety and depressive symptoms in patients with conversion disorder. $J$ Coll Physicians Surg Pak. 2005;15(8):489-492.

12. Mahler C, Macqueen G, Samaan Z. A postmenopausal woman presenting with Ekbom syndrome associated with recurrent depressive disorder: a case report. Cases J. 2008;1(1):54.

13. Robinson BF, Epstein SE, Beiser DG, Braunwald E. Control of heart rate by the autonomic nervous system. Studies in man on the interrelation between baroreceptor mechanisms and exercise. Circ Res.1966;19(2): 400-411.

14. Kannel WB, Kannel C, Paffenbarger RS Jr, Cupples AL. Heart rate and cardiovascular mortality: The Framingham Study. Am Heart J. 1987;113(6):1489-1494.

15. Gillum RF, Makuc DM, Feldman JJ. Pulse rate, coronary heart disease, and death: the NHANES I Epidemiologic Follow-up Study. Am Heart J. 1991;121(1 Pt 1):172-177.

16. Greenland P, Daviglus ML, Dyer AR, et al. Resting heart rate is a risk factor for cardiovascular and noncardiovascular mortality: the Chicago Heart Association Detection Project in Industry. Am J Epidemiol. 1999;149(9):853-862.

17. Seccareccia F, Pannozzo F, Dima F, et al; Malattie Cardiovascolari Aterosclerotiche Istituto Superiore di Sanita Project. Heart rate as a predictor of mortality: the MATISS project. Am J Public Health. 2001; 91(8):1258-1263.

18. Grosgogeat Y, Ducardonnet A, Valty J, et al. Physiological limits of variations in heart rate measured by the Holter method in 134 normal subjects. Arch Mal Coeur Vaiss. 1986;79(3):324-331. French.

19. Goldstein IB, Jamner LD, Shapiro D. Ambulatory blood pressure and heart rate in healthy male paramedics during a workday and a nonworkday. Health Psychol. 1992;11(1):48-54.

20. Degaute JP, van de Borne P, Linkowski P, Van Cauter E. Quantitative analysis of the 24-hour blood pressure and heart rate patterns in young men. Hypertension. 1991;18(2):199-210.

21. Nelson W, Tong YL, Lee JK, Halberg F. Methods for cosinorrhythmometry. Chronobiologia. 1979;6(4):305-323.
ChronoPhysiology and Therapy

\section{Publish your work in this journal}

ChronoPhysiology and Therapy is an international, peer-reviewed, open access journal focusing on research into the cyclic variations and rhythmicity in physiological processes in the body and the research and development and optimal timing of administration of therapeutic targets to achieve improved outcomes and quality of life for the patient. The

\section{Dovepress}

manuscript management system is completely online and includes a very quick and fair peer-review system. Visit http://www.dovepress.com/ testimonials.php to read real quotes from published authors. 